\title{
INTEGRABLE CONDITIONS FOR DIRAC EQUATION AND SCHRÖDINGER EQUATION
}

\section{YING-QIU GU}

School of Mathematical Science

Fudan University

Shanghai 200433

P. R. China

e-mail: yqgu@fudan.edu.cn

\begin{abstract}
By constructing the commutative operators chain, we derive the integrable conditions for solving the eigenfunctions of Dirac equation and Schrödinger equation. These commutative relations correspond to the intrinsic symmetry of the physical system, which are equivalent to the original partial differential equation can be solved by separation of variables. Detailed calculation shows that, only a few cases can be completely solved by separation of variables. In general cases, we have to solve the Dirac equation and the Schrödinger equation by effective perturbation or approximation methods, especially in the cases including nonlinear potential or self-interactive potentials.
\end{abstract}

\section{Introduction}

Fermions with spin $-\frac{1}{2}$ are described by Dirac equations. Many physicists such as H. Weyl, W. Heisenberg, once proposed using nonlinear spinor equations to establish unified field theory for elementary particles

2020 Mathematics Subject Classification: 13P25, 17B81, 35Q40, 37K10, 70H06, 81R12.

Keywords and phrases: eigenfunction, commutative relation, separation of variables, nonlinear Dirac equation, Abelian Lie algebra, Clifford algebra.

Received March 13, 2020; Revised April 13, 2020.

(C) 2020 Scientific Advances Publishers 
$[1,2]$. Some rigorous solutions for the simplest dark nonlinear spinor models were obtained and analyzed [3]-[11]. The nonlinear spinor coupling with self-electromagnetic field was calculated in [12]-[15], but only roughly approximate results were obtained due to the complexity of the interaction. In contrast, for the linear Schrödinger equation, Pauli equation and Dirac equation in spherical coordinate system $(r, \theta, \varphi)$ coupling with external potential $V(r)$ and $\vec{A}(r, \theta)$, there are a lot of rigorous eigen solutions obtained by separation of variables [16]-[29]. Some theoretical analyses on the existence of stationary solutions to nonlinear Dirac equations were provided in [30]-[36].

In the resolution of eigenfunctions to the linear Dirac equation, we find that the commutative relations [16]

$$
\left[\hat{H}, \hat{J}_{z}\right]=0, \quad[\hat{H}, \hat{K}]=0, \quad\left[\hat{K}, \hat{J}_{z}\right]=0
$$

play an important role, where the operators $\left(\hat{J}_{z}, \hat{K}, \hat{H}\right)$ stand for angular momentum, spin-orbit coupling, and total energy operators, respectively. They are good quantum numbers guaranteeing the common eigenfunctions exist. Noticing the definition of operators chain

$$
\hat{J}_{z}=J_{z}\left(\partial_{\varphi}\right), \quad \hat{K}=K\left(\partial_{\varphi}, \partial_{\theta}\right), \quad \hat{H}=H\left(\partial_{\varphi}, \partial_{\theta}, \partial_{r}\right),
$$

which enable us to solve the eigen solutions by separation of variables, and then the original problem reduces to ordinary differential equations. So the existence of complete commutative operator chain forms the integrable condition for Dirac equation or Schrödinger equation. Since the construction of commutative operator chain is quite simple and elementary, and the existence conditions are easily converted into some algebraic requirements for potentials as shown below, then the integrable condition of a quantum system is equivalently obtained. 
Denote the Minkowski metric by $\eta_{\mu \nu}=\operatorname{diag}[1,-1,-1,-1]$, where $\mu, \nu \in\{0,1,2,3\}$. Pauli matrices by

$$
\vec{\sigma}=\left(\sigma^{j}\right)=\left\{\left(\begin{array}{ll}
0 & 1 \\
1 & 0
\end{array}\right), \quad\left(\begin{array}{cc}
0 & -i \\
i & 0
\end{array}\right), \quad\left(\begin{array}{rr}
1 & 0 \\
0 & -1
\end{array}\right)\right\}, \quad j \in\{1,2,3\} .
$$

Define $4 \times 4$ Hermitian matrices as follows:

$$
\alpha^{\mu}=\left\{\left(\begin{array}{ll}
I & 0 \\
0 & I
\end{array}\right), \quad\left(\begin{array}{rr}
0 & \vec{\sigma} \\
\vec{\sigma} & 0
\end{array}\right)\right\}, \quad \gamma=\left(\begin{array}{rr}
I & 0 \\
0 & -I
\end{array}\right), \quad \beta=\left(\begin{array}{rr}
0 & -i I \\
i I & 0
\end{array}\right) .
$$

In this paper, we adopt the Hermitian matrices (1.4) instead of Dirac matrices $\gamma^{\mu}$ for convenience of calculation.

The basic problem in this paper is to examine a nonlinear spinor field $\phi$ moving in 4 -vector potential $A^{\mu}$. The corresponding Lagrangian is generally given by [13]

$$
\mathcal{L}=\phi^{+}\left[\alpha^{\mu}\left(i \hbar \partial_{\mu}-e A_{\mu}\right)-m c \gamma\right] \phi+F(\check{\gamma}, \check{\beta}),
$$

where $m>0$ is a constant mass, the index ' + ' stands for transpose conjugation, $F$ is the nonlinear coupling potential such as $F=\frac{1}{2} \omega_{\gamma^{2}}^{2}$, which is usually the polynomial of scalar $\check{\gamma}$ and pseudo-scalar $\breve{\beta}$ defined by

$$
\check{\gamma}=\phi^{+} \gamma \phi, \quad \check{\beta}=\phi^{+} \beta \phi .
$$

The variation of (1.5) with respect to $\phi^{+}$gives the dynamic equation

$$
i \hbar \partial_{0} \phi=\hat{H} \phi, \quad \hat{H} \equiv \vec{\alpha} \cdot(-i \hbar \nabla-e \vec{A})+e A_{0}+\left(m c-F_{\gamma}\right) \gamma-F_{\beta} \beta,
$$

where $F_{\gamma}=\frac{\partial F}{\partial \check{\gamma}}$ and $F_{\beta}=\frac{\partial F}{\partial \check{\beta}}$. 
Let coordinate $x^{3}=z$ along the direction of magnetic field $\vec{B}$, then we locally have $[8,9,13]$

$$
\vec{A}=A(r, \theta)(-\sin \varphi, \cos \varphi, 0),
$$

which satisfies the Coulomb gauge $\nabla \cdot \vec{A}=0$. In the spherical coordinate system $(r, \theta, \varphi)$, we have

$$
\vec{\sigma} \cdot \nabla=\sigma_{r} \partial_{r}+\frac{1}{r} \sigma_{\theta} \partial_{\theta}+\frac{1}{r \sin \theta} \sigma_{\varphi} \partial_{\varphi}
$$

where $\left(\sigma_{r}, \sigma_{\theta}, \sigma_{\varphi}\right)$ is given by

$$
\left\{\left(\begin{array}{cc}
\cos \theta & \sin \theta e^{-\varphi i} \\
\sin \theta e^{\varphi i} & -\cos \theta
\end{array}\right),\left(\begin{array}{cc}
\sin \theta & \cos \theta e^{-\varphi i} \\
\cos \theta e^{\varphi i} & \sin \theta
\end{array}\right),\left(\begin{array}{cc}
0 & i e^{-\varphi i} \\
i e^{\varphi i} & 0
\end{array}\right)\right\} .
$$

Let $\hat{J}$ be the angular momentum operator for the spinor field, we have

$$
\hat{J}=\vec{r} \times \hat{p}+\frac{1}{2} \hbar \vec{S}, \quad \hat{p}=-i \hbar \nabla, \quad \vec{S}=\operatorname{diag}(\vec{\sigma}, \vec{\sigma}) .
$$

However, it should be pointed out that, here $\hat{p}=-i \hbar \nabla$ is only geometrical momentum rather the physical one $\hat{p}=-i \hbar \nabla-e \vec{A}$. Then the angular momentum $\hat{J}_{3}=\hat{J}_{z}$ commutates with the nonlinear Hamilton operator (1.7), and the eigenfunctions of $\hat{J}_{z}=-i \hbar \partial_{\varphi}+\frac{1}{2} \hbar S_{3}$ are equivalent to the following form:

$$
\phi=\left(u_{1}, u_{2} e^{\varphi i},-i v_{1},-i v_{2} e^{\varphi i}\right)^{T} \exp \left(\kappa \varphi i-\frac{m c^{2}}{\hbar} t i\right)
$$

with $(\kappa=0, \pm 1, \pm 2, \ldots)$, where $u_{k}, v_{k}(k=1,2)$ are real functions of $(r, \theta)$ but independent on $\varphi$ and $t$, the index $T$ stands for transposed matrix. 


\section{Commutative Algebras for Dirac Equation}

In spherical coordinate system, we have the following explicit operator relations [16]. For orbit angular momentum operator $\hat{L}=\vec{r} \times \hat{p}$, we have

$$
\begin{aligned}
& \hat{L}_{x}=i \hbar\left(\sin \varphi \partial_{\theta}+\cot \theta \cos \varphi \partial_{\varphi}\right), \\
& \hat{L}_{y}=i \hbar\left(-\cos \varphi \partial_{\theta}+\cot \theta \sin \varphi \partial_{\varphi}\right), \\
& \hat{L}_{z}=-i \hbar \partial_{\varphi}, \\
& \hat{L}^{2}=-\hbar^{2}\left(\partial_{\theta}^{2}+\cot \theta \partial_{\theta}+\frac{1}{\sin \theta^{2}} \partial_{\varphi}^{2}\right) .
\end{aligned}
$$

For the nonlinear equation (1.7), detailed calculation shows that

$$
\left[\hat{H}, \hat{J}_{z}\right]=0 .
$$

(2.5) means $\hat{J}_{z}$ is still a good quantum number for the nonlinear Dirac equation. But we have $\left[\hat{H}, \hat{J}_{x}\right] \neq 0$ and $\left[\hat{H}, \hat{J}_{y}\right] \neq 0$. Define the spinorbit coupling operator by

$$
\hat{K} \equiv \gamma(\hat{L} \cdot \vec{S}+\hbar)=K_{0}-i \hbar\left(K_{\theta} \partial_{\theta}+K_{\varphi} \partial_{\varphi}\right), \quad K_{0} \equiv \hbar \gamma,
$$

where $K_{\theta}$ and $K_{\varphi}$ defined by

$$
\begin{aligned}
& K_{\theta}=\operatorname{diag}\left[\left(\begin{array}{cc}
0 & -i e^{-\varphi i} \\
i e^{\varphi i} & 0
\end{array}\right),\left(\begin{array}{cc}
0 & i e^{-\varphi i} \\
-i e^{\varphi i} & 0
\end{array}\right)\right], \\
& K_{\varphi}=\operatorname{diag}\left[\left(\begin{array}{cc}
1 & -\cot \theta e^{-\varphi i} \\
-\cot \theta e^{\varphi i} & -1
\end{array}\right),\left(\begin{array}{cc}
-1 & \cot \theta e^{-\varphi i} \\
\cot \theta e^{\varphi i} & 1
\end{array}\right)\right],
\end{aligned}
$$

then for (1.7), we can check

$$
[\hat{H}, \hat{K}]=K_{V}+K_{A}+K_{\beta},
$$


in which

$$
\begin{aligned}
K_{V}= & -i \hbar\left(e \partial_{\theta} V-\partial_{\theta} F_{\gamma} \gamma\right) \gamma\left(\sin \varphi S_{1}-\cos \varphi S_{2}\right), \\
K_{A}= & -e \hbar\left(A \gamma\left(\sin \varphi \alpha^{1}-\cos \varphi \alpha^{2}\right)-\left(\partial_{\theta} A+\cot \theta A+2 A \partial_{\theta}\right) \beta\right), \\
K_{\beta}= & 2 \hbar \gamma \beta F_{\beta}+\hbar\left(\partial_{\theta} F_{\beta}+2 F_{\beta} \partial_{\theta}\right)\left(\sin \varphi \alpha^{1}-\cos \varphi \alpha^{2}\right) \\
& +2 \hbar F_{\beta}\left(\cot \theta\left(\cos \varphi \alpha^{1}+\sin \varphi \alpha^{2}\right)-\alpha^{3}\right) \partial_{\varphi} .
\end{aligned}
$$

Relations (2.9)-(2.12) reflect the influence of parameters on the integrability.

\section{Conditions for Separation of Variables}

For the nonlinear Hamiltonian (1.7), the commutative relation (2.5) shows $\hat{J}_{z}$ is still a good quantum number, but (2.9) shows $\hat{K}$ is not. In what follows, we look for a new operator $\hat{T}$, such that the systems (1.7) can be solved by separation of variables. This is equivalent to the

existence of operator $\hat{T}=T\left(\partial_{\theta}, \partial_{\varphi}\right)$ or $\hat{T}^{\prime}=T^{\prime}\left(\partial_{r}, \partial_{\varphi}\right)$, which satisfies commutative relations

$$
\left[\hat{J}_{z}, \hat{T}\right]=0, \quad[\hat{H}, \hat{T}]=0 .
$$

We only consider the following first order operator

$$
\hat{T}=T_{0}-i \hbar\left(T_{\theta} \partial_{\theta}+T_{\varphi} \partial_{\varphi}\right),
$$

where $\left(T_{0}, T_{\theta}, T_{\varphi}\right)$ are all Hermitian matrices, and their components are smooth functions of $(r, \theta, \varphi)$. For Dirac equation, it is enough to look for the linear operators similar to (3.2). But for the Schrödinger and Pauli equations, we should consider the second order operator similar to $\hat{L}^{2}$ as shown below. Different from $\hat{p}$ and $\hat{J}$, the operators constructed from the procedure may have not manifest physical meanings. 
According to condition $\left[\hat{J}_{z}, \hat{T}\right]=0$, we can solve

$$
T_{0}=\left(\begin{array}{cccc}
P_{11} & P_{12} \cot \theta e^{-i \phi} & P_{13} & P_{14} \cot \theta e^{-i \phi} \\
P_{21} \cot \theta e^{i \phi} & P_{22} & P_{23} \cot \theta e^{i \phi} & P_{24} \\
P_{31} & P_{32} \cot \theta e^{-i \phi} & P_{33} & P_{34} \cot \theta e^{-i \phi} \\
P_{41} \cot \theta e^{i \phi} & P_{42} & P_{43} \cot \theta e^{i \phi} & P_{44}
\end{array}\right),
$$

$$
\begin{gathered}
T_{\theta}=\left(\begin{array}{cccc}
M_{11} & M_{12} e^{-i \phi} & M_{13} & M_{14} e^{-i \phi} \\
M_{21} e^{i \phi} & M_{22} & M_{23} e^{i \phi} & M_{24} \\
M_{31} & M_{32} e^{-i \phi} & M_{33} & M_{34} e^{-i \phi} \\
M_{41} e^{i \phi} & M_{42} & M_{43} e^{i \phi} & M_{44}
\end{array}\right), \\
T_{\varphi}=\left(\begin{array}{cccc}
N_{11} & N_{12} \cot \theta e^{-i \phi} & N_{13} & N_{14} \cot \theta e^{-i \phi} \\
N_{21} \cot \theta e^{i \phi} & N_{22} & N_{23} \cot \theta e^{i \phi} & N_{24} \\
N_{31} & N_{32} \cot \theta e^{-i \phi} & N_{33} & N_{34} \cot \theta e^{-i \phi} \\
N_{41} \cot \theta e^{i \phi} & N_{42} & N_{43} \cot \theta e^{i \phi} & N_{44}
\end{array}\right),
\end{gathered}
$$

where $\left(P_{k l}, M_{k l}, N_{k l}\right)$ are functions of $(r, \theta)$, the factor 'cot $\theta$ ' is introduced for convenience of the following calculation.

The relation $[\hat{H}, \hat{T}]$ can be expressed as

$$
\begin{aligned}
{[\hat{H}, \hat{T}]=} & H_{\theta \theta} \partial_{\theta}^{2}+H_{r \theta} \partial_{r \theta}+\left(H_{\theta}+H_{\theta \varphi} \partial_{\varphi}\right) \partial_{\theta}+\left(H_{r}+H_{r \varphi} \partial_{\varphi}\right) \partial_{r} \\
& +\left(H_{0}+H_{\varphi} \partial_{\varphi}+H_{\varphi \varphi} \partial_{\varphi}^{2}\right),
\end{aligned}
$$


where the coefficient matrices can be obtained by straightforward calculation. $\left(H_{\theta \theta}, H_{r \theta}, H_{\theta \varphi}, H_{r \varphi}, H_{\varphi \varphi}\right)$ are only functions of $\left(P_{k l}, M_{k l}, N_{k l}\right)$, but the others depend on their first order derivatives. The operator $\partial_{\varphi}$ should be replaced by matrix $D_{\varphi}$ due to solution (1.12),

$$
\begin{gathered}
\partial_{\varphi} \leftrightarrow D_{\varphi}=i \operatorname{diag}(\kappa, \kappa+1, \kappa, \kappa+1), \\
\partial_{\varphi}^{2} \leftrightarrow D_{\varphi}^{2}=-\operatorname{diag}\left(\kappa^{2},(\kappa+1)^{2}, \kappa^{2},(\kappa+1)^{2}\right) .
\end{gathered}
$$

Then $[\hat{H}, \hat{T}]=0$ is equivalent to the following equations:

$$
\begin{gathered}
H_{\theta \theta}=H_{r \theta}=0, \\
H_{\theta}+H_{\theta \varphi} D_{\varphi}=0, \\
H_{r}+H_{r \varphi} D_{\varphi}=0, \\
H_{0}+H_{\varphi} D_{\varphi}+H_{\varphi \varphi} D_{\varphi}^{2}=0 .
\end{gathered}
$$

The differential equations including in (3.9)-(3.12) are linear, and one component usually satisfies two independent equations, which lead to constant solutions. So they can be easily solved as shown below.

By (3.9), we can solve

$$
T_{\theta}=\left(\begin{array}{cccc}
W_{1} & -i W_{2} e^{-i \phi} & W_{3} & W_{4} e^{-i \phi} \\
i W_{2} e^{i \phi} & W_{1} & -W_{4} e^{i \phi} & W_{3} \\
W_{3} & -W_{4} e^{-i \phi} & W_{1} & i W_{2} e^{-i \phi} \\
W_{4} e^{i \phi} & W_{3} & -i W_{2} e^{i \phi} & W_{1}
\end{array}\right),
$$

in which $W_{k}=W_{k}(r, \theta, \kappa),(k=1,2,3,4)$. Again by (3.10), we find that $W_{1}=W_{3}=W_{4}=0$, and $W_{2} \neq 0$ is a constant. Obviously $\hat{T}$ can be only 
determined to a constant factor and constant translation, so we choose $W_{2}=1$ to fix the solution. Then in equivalent sense, we have

$$
T_{\theta}=K_{\theta}
$$

For the other equations in (3.10)-(3.12), detailed calculation shows that the solutions for $P_{k l}$ and $N_{k l}$ are underdetermined. Expressing $P_{k l}$ by $N_{k l}$, we can get the following results by simple calculation:

$$
\begin{gathered}
V=V(r), \quad A=r^{-1} U(\theta), \quad \partial_{\theta} F_{\gamma}=0, \quad F_{\beta}=0, \\
T_{0}-i \hbar T_{\varphi} D_{\varphi}=\left(K_{0}-i \hbar K_{\varphi} D_{\varphi}\right)+e \hbar U \operatorname{diag}\left(\sigma_{\theta},-\sigma_{\theta}\right)+\lambda_{0},
\end{gathered}
$$

where $\lambda_{0}$ is a constant. If we set $\hat{T}=\hat{K}$ when $U=0$, we get $\lambda_{0}=0$. Substituting $D_{\varphi} \rightarrow \partial_{\varphi}$ into (3.16), in equivalent sense, we finally get

$$
T_{0}=K_{0}+e \hbar U \operatorname{diag}\left(\sigma_{\theta},-\sigma_{\theta}\right), \quad T_{\theta}=K_{\theta}, \quad T_{\varphi}=K_{\varphi} .
$$

(3.17) shows $\hat{T}$ is almost the spin-orbit coupling term $\hat{K}$. Only if the vector potential $\vec{A}$ takes some special shape, we can exactly solve the Dirac equation. The physical meanings of the additional term $e \hbar U$ diag $\left(\sigma_{\theta},-\sigma_{\theta}\right)$ should be clarified by Clifford algebra as shown below.

In the spherical coordinate system, the above procedure is invertible, so Equations (3.15) actually form the sufficient and necessary conditions of the separation of variables to Dirac equation with axisymmetry. Noticing the relation (3.17), conditions (3.15) can also be verified by the commutative relations (2.9)-(2.12). The following cases were studied in previous works by many researchers: $\left\{V=A=F_{\beta}=\partial_{\theta} F_{\gamma}=0\right\}$ is the simplest nonlinear case discussed in [3]-[9]. $\left\{V=V(r), A=F_{\beta}=0, F_{\gamma}=G(r)\right\}$ are the cases solved in [16] and [17]-[28]. $\left\{V=\frac{Z e}{r}, A=\frac{e}{r \sin \theta}(a-b \cos \theta), F_{\beta}=F_{\gamma}=0\right\}$ is the case solved in [29]. 
For the general case of (3.15), although the separation of variables is valid for the Dirac equation (1.7), but the solutions to the reduced ordinary differential equations usually can not be expressed by elementary functions. So some auxiliary numerical computation is still necessary.

For the Schrödinger equation

$$
i \hbar \partial_{0} \phi=\hat{H} \phi, \quad \hat{H} \equiv-\frac{\hbar^{2}}{2 m}\left(\partial_{r}^{2}+\frac{2}{r} \partial_{r}+\frac{1}{\hbar^{2} r^{2}} \hat{L}^{2}\right)+V(r, \theta),
$$

the calculation is simple. Obviously, for $\hat{L}_{z}=-\hbar i \partial_{\varphi}$, we have

$$
\left[\hat{H}, \partial_{\varphi}\right]=0, \quad \partial_{\varphi} \leftrightarrow \kappa i
$$

We look for the following second order operator:

$$
\hat{Y}(\theta, \varphi)=Y_{0}+Y_{\theta} \partial_{\theta}+\hat{L}^{2} .
$$

By $\left[\hat{Y}, \partial_{\varphi}\right]=0$, we get

$$
\partial_{\varphi} Y_{0}=\partial_{\varphi} Y_{\theta}=0
$$

By $[\hat{H}, \hat{Y}]=0$, we find

$$
\begin{gathered}
V=W(r)+\frac{1}{r^{2}} U(\theta), \\
Y_{0}=\lambda_{0}-2 m U(\theta), \quad Y_{\theta}=0,
\end{gathered}
$$

where $U(\theta)$ and $W(r)$ are arbitrary functions. (3.22) is the condition to solve the eigenfunctions of (3.18) by separating variables. Different from the Dirac equation, (3.22) shows $V$ can vary with $\theta$. 


\section{The Relations in the Formalism of Clifford Algebra}

The above discussion is based on the conventional notations of quantum mechanics. Now we make some general considerations. We express the main contents in the formalism of Clifford algebra, because this algebra faithfully and exactly reflects the intrinsic symmetry and property of space-time and fields [10, 37]. In this formalism, the calculations such as (3.3)-(3.6) become much simple and clear, and we may get some

new insights into the symmetries of the integrable conditions for the Dirac equations.

Denote the generators of $C \ell(1,3)$ by

$$
\begin{gathered}
\gamma^{a}=\left(\begin{array}{cc}
0 & \widetilde{\sigma}^{a} \\
\sigma^{a} & 0
\end{array}\right), \quad \gamma^{5}=\left(\begin{array}{cc}
I & 0 \\
0 & -I
\end{array}\right), \\
\sigma^{a}=(I, \vec{\sigma}), \quad \widetilde{\sigma}^{a}=\left(\sigma^{0},-\vec{\sigma}\right), \quad \vec{\sigma}=\left(\sigma^{1}, \sigma^{2}, \sigma^{3}\right) .
\end{gathered}
$$

For dynamics (1.7), making transformation

$$
\phi^{\prime}=C \phi, \quad C=\frac{1}{\sqrt{2}}\left(\gamma^{0}+\gamma^{5}\right)=C^{-1},
$$

we get Dirac equation with coefficient in standard Clifford algebra as follows:

$$
\gamma^{a}\left(i \hbar \partial_{a}-e A_{a}\right) \phi^{\prime}+i \gamma^{5} F_{\beta} \phi^{\prime}=\left(m c-F_{\gamma}\right) \phi^{\prime}
$$

The quadratic scalar and pseudo scalar become

$$
\check{\gamma}=\phi^{+} \gamma^{0} \phi^{\prime}, \quad \check{\beta}=\phi^{\prime+} i \gamma^{0} \gamma^{5} \phi^{\prime}=\phi^{\prime+} \beta \phi^{\prime} .
$$

By transformation (4.3), we get

$$
\hat{T}^{\prime}=\gamma^{0} C \hat{T} C^{-1}=\hat{K}^{\prime}+e \hbar U S^{\theta} .
$$

(4.6) shows $\hat{T}$ includes a spin-potential coupling term eћrAS ${ }^{\theta}$, which reflects the spin-vector potential interaction. Its physical meaning can be clarified only by Clifford algebra. In what follows we directly use $\phi$ to replace $\phi^{\prime}$ for simplicity. 
To get rigorous solution of Dirac equation, using coordinate system reflects the symmetry of the interaction potential is important. The results derived above are only relevant to the potentials near the spherical symmetry. In general cases, we should discuss the problem in curved space-time. Clifford algebra is the perfect language. Then Dirac equation (4.4) becomes [10,37,38]

$$
\gamma^{\mu}\left(\hat{p}_{\mu}+\frac{1}{2} \hbar \gamma^{5} \Omega_{\mu}\right) \phi+i \gamma^{5} F_{\beta} \phi=\left(m c-F_{\gamma}\right) \phi
$$

in which the coefficient belongs to $\Lambda^{0} \cup \Lambda^{1} \cup \Lambda^{3} \cup \Lambda^{4} \subset C \ell(1,3)$, and parameters and operators are defined as

$$
\begin{gathered}
\gamma^{\mu}=h_{a}^{\mu} \gamma^{a}, \quad \gamma_{\mu}=l_{\mu}^{a} \gamma_{a}, \quad \gamma_{\mu} \gamma_{\nu}+\gamma_{\nu} \gamma_{\mu}=2 g_{\mu \nu}, \\
\hat{p}_{\mu}=i \hbar\left(\partial_{\mu}+\Upsilon_{\mu}\right)-e A_{\mu}, \quad S_{a b}^{\mu \nu} \equiv \frac{1}{2}\left(h^{\mu}{ }_{a} h_{b}^{\nu}{ }_{b} h^{\nu}{ }_{a} h_{b}^{\mu}\right) \operatorname{sgn}(a-b), \\
\Upsilon_{\mu}=\frac{1}{2} h_{a}^{\nu}\left(\partial_{\mu} l_{\nu}^{a}-\partial_{\nu} l_{\mu}^{a}\right), \quad \Omega^{\alpha}=\frac{1}{4} \epsilon^{d a b c} h_{d}^{\alpha} h_{a}^{\beta} S_{b c}^{\mu \nu} \partial_{\beta} g_{\mu \nu} .
\end{gathered}
$$

Here we use Greek index stands for curved space-time, Latin index for Minkowski space-time, and $(j, k, l, m, n)$ for spatial indices. If the metric can be diagonalized, we have $\Omega_{\mu} \equiv 0$.

Obviously, for eigenstates of spinor, the time $t$ is special, which should be global simultaneity. That is to say, we must discuss the Hamiltonian formalism of (4.7) in the natural coordinate system $d s^{2}=g_{00} d t^{2}-g_{k l} d x^{k} d x^{l}$ with global realistic time [39] or in local Gauss normal coordinate system (LGCS) $d s^{2}=d t^{2}-g_{k l} d x^{k} d x^{l}$. We take LGCS for simplicity.

In LGCS, to lift and lower the index of a vector means

$$
\Upsilon^{0}=\Upsilon_{0}=\frac{1}{2} \partial_{t}(\ln \sqrt{g}), \quad \Upsilon^{k}=-g^{k} \Upsilon_{l}, \quad g_{k l} g^{l n}=\delta_{k}^{n}
$$


Then (4.7) can be rewritten in the following Hamiltonian formalism:

$$
i\left(\partial_{t}+\Upsilon_{t}\right) \phi=\hat{H} \phi, \quad \hat{H}=-\alpha^{k} \hat{p}_{k}+e A_{0}+\left(m c-F_{\gamma}\right) \gamma^{0}-\beta F_{\beta}-\Omega_{\mu} \hat{S}^{\mu},
$$

where $\hat{H}$ is the Hamiltonian of dynamics in curved space-time, and $\left(\alpha^{\mu}, \hat{S}^{\mu}\right)$ are respectively current and spin operators defined by

$$
\alpha^{\mu} \equiv \gamma^{0} \gamma^{\mu}=h^{\mu}{ }_{a} \operatorname{diag}\left(\sigma^{a}, \widetilde{\sigma}^{a}\right), \quad \hat{S}^{\mu} \equiv \frac{1}{2} \hbar \alpha^{\mu} \gamma^{5}=\frac{1}{2} \hbar h^{\mu}{ }_{a} \operatorname{diag}\left(\sigma^{a},-\widetilde{\sigma}^{a}\right) .
$$

$\vec{S} \in \Lambda^{3}$ is the usual spin of Dirac bispinor. The eigenstate of energy means $i\left(\partial_{t}+\Upsilon_{t}\right) \phi=E \phi$.

The Hamiltonian $\hat{H}$ has the following properties:

(1) $\hat{H}$ is Hermitian, so its eigen values are all real.

(2) $\hat{H}$ is a linear operator with respect to derivatives $\nabla_{k}=\partial_{k}+\Upsilon_{k}$.

(3) $\hat{H} \in C \ell(4,0)$, and the generators of $C \ell(4,0)$ are $\left(\gamma^{0}, \alpha^{k}\right)$.

(4) $\hat{H}$ does not include component in $\Lambda^{2}$, i.e., we have not $\gamma^{\alpha \beta}$ term in $\hat{H}$.

From the calculations in the above sections, we learn that, to solve the rigorous eigen solution to (4.12) is equivalent to construct an Abelian Lie algebra $\left\{H_{1}, H_{2}, H_{3}=\hat{H}\right\}$, where $\forall H_{k}$ are linear independent. Besides, $\forall H_{k}$ should satisfy all listed properties of $\hat{H}$. Under these constraints, we can solve the other two operators $\left(H_{1}, H_{2}\right)$ for any dynamics of spinor in principle. And then, by a linear transformation with respect to derivatives $\nabla_{k}$, we can get the Abelian Lie group with proper elements $\left\{\hat{H}_{1}\left(\nabla_{1}\right), \hat{H}_{2}\left(\nabla_{1}, \nabla_{2}\right), \hat{H}\right\}$. Thus the solvability of Dirac 
equation is thoroughly resolved in theory. Obviously this procedure can be generalized for high dimensional problems. However, to derive the elements of Abelian Lie group $\left\{\hat{H}_{1}, \hat{H}_{2}\right\}$ for a concrete problem is not an easy task. It should be realized by computing program.

\section{Discussion and Conclusion}

From the above calculation, we find the method is quite simple and direct. We get the following conclusion and remarks. The above procedure has general significance to solve the rigorous eigen solution of Dirac equation. The separation of variables is equivalent to the existence of the following Hermitian operators chain:

$$
\hat{H}_{1}=H_{1}\left(\partial_{1}\right), \quad \hat{H}_{2}=H_{2}\left(\partial_{1}, \partial_{2}\right), \ldots, \quad \hat{H}_{n}=H_{n}\left(\partial_{1}, \partial_{2}, \ldots, \partial_{n}\right),
$$

which form an Abelian Lie algebra

$$
\left[\hat{H}_{j}, \hat{H}_{k}\right]=0, \quad(j, k=1, \ldots, n) .
$$

(5.2) forms the integrable conditions of the equation, and can be obtained via simple calculation. In general, a complete chain similarly to the case of (1.7) may be absent, but the incomplete chain is still helpful to simplify the dynamic equation.

The commutative relations (5.2) are conditional relations, that is, it is enough to hold only for the eigenfunctions, rather than hold in the whole function space of the solutions. The solution of the nonlinear spinors is an example [3]-[9]. So we can use the information of solutions for solving the operators chain. By Clifford algebra, we can consider the problems in curved space-time. 


\section{References}

[1] H. Weyl, A remark on the coupling of gravitation and electron, Physical Review 77(5) (1950), 699-701.

DOI: https://doi.org/10.1103/PhysRev.77.699

[2] W. Heisenberg, Doubts and hopes in quantum electrodynamics, Physica 19(1-12) (1953), 897-908.

DOI: https://doi.org/10.1016/S0031-8914(53)80100-X

[3] R. Finkelstein, R. LeLevier and M. Ruderman, Nonlinear spinor fields, Physical Review 83(2) (1951), 326-332.

DOI: https://doi.org/10.1103/PhysRev.83.326

[4] M. Soler, Classical, stable, nonlinear spinor field with positive rest energy, Physical Review D 1(10) (1970), 2766-2767.

DOI: https://doi.org/10.1103/PhysRevD.1.2766

[5] K. Johnson, A field theory Lagrangian for the MIT bag model, Physics Letters B 78(2-3) (1978), 259-262.

DOI: https://doi.org/10.1016/0370-2693(78)90018-7

[6] P. Mathieu and R. Saly, Baglike solutions of a Dirac equation with fractional nonlinearity, Physical Review D 29(12) (1984), 2879-2883.

DOI: https://doi.org/10.1103/PhysRevD.29.2879

[7] A. F. Ranada, Classical Nonlinear Dirac Field Models of Extended Particles, In: Quantum Theory, Groups, Fields and Particles, Edited by A. O. Barut, Amsterdam, Reidel, 1983.

[8] Y. Q. Gu, Some properties of the spinor soliton, Advances in Applied Clifford Algebras 8(1) (1998), 17-29.

\section{DOI: https://doi.org/10.1007/BF03041923}

[9] Y. Q. Gu, Characteristic functions and typical values of the nonlinear dark spinor, arXiv:hep-th/0611210.

[10] Y. Q. Gu, Clifford algebra, Lorentz transformation and unified field theory, Advances in Applied Clifford Algebras 28(2) (2018); Article 37.

DOI: https://doi.org/10.1007/s00006-018-0852-0

[11] W. Fushchych and R. Zhdanov, Symmetries and Exact Solutions of Nonlinear Dirac Equations, Kyiv Mathematical Ukraina Publisher, Ukraine (1997); arXiv: math$\mathrm{ph} / 0609052$.

[12] M. Wakano, Intensely localized solutions of the classical Dirac-Maxwell field equations, Progress of Theoretical Physics 35(6) (1966), 1117-1141.

DOI: https://doi.org/10.1143/PTP.35.1117 
[13] Y. Q. Gu, Spinor soliton with electromagnetic field, Advances in Applied Clifford Algebras 8(2) (1998), 271-282.

DOI: https://doi.org/10.1007/BF03043099

[14] Y. Q. Gu, The electromagnetic potential among nonrelativistic electrons, Advances in Applied Clifford Algebras 9(1) (1999), 55-60.

DOI: https://doi.org/10.1007/BF03041937

[15] Y. Q. Gu, New approach to $N$-body relativistic quantum mechanics, International Journal of Modern Physics A 22(11) (2007), 2007-2019.

DOI: https://doi.org/10.1142/S0217751X07036233

[16] W. Greiner, Relativistic Quantum Mechanics: Wave Equations, Springer-Verlag Berlin Heidelberg, 1990.

\section{DOI: https://doi.org/10.1007/978-3-662-04275-5}

[17] G. V. Shishkin and V. M. Villalba, Electrically neutral Dirac particles in the presence of external fields: Exact solutions, Journal of Mathematical Physics 34(11) (1993), 5037-5049.

DOI: https://doi.org/10.1063/1.530340

[18] V. G. Bagrov, M. C. Baldiotti, D. M. Gitman and I. V. Shirokov, New solutions of relativistic wave equations in magnetic fields and longitudinal fields, Journal of Mathematical Physics 43(5) (2002), 2284-2305.

DOI: https://doi.org/10.1063/1.1461428

[19] V. M. Villalba, The angular momentum operator in the Dirac equation, European Journal of Physics 15(4) (1994), 191-196.

DOI: https://doi.org/10.1088/0143-0807/15/4/006

[20] M. A. Rodríguez and P. Winternitz, Quantum superintegrability and exact solvability in $n$ dimensions, Journal of Mathematical Physics 43(3) (2002), 1309-1322.

DOI: https://doi.org/10.1063/1.1435077

[21] S. H. Dong, The Dirac equation with a Coulomb potential in D dimensions, Journal of Physics A: Mathematical and General 36(18) (2003), 1-10.

DOI: https://doi.org/10.1088/0305-4470/36/18/303

[22] X. Y. Gu, Z. Q. Ma and S. H. Dong, Exact solutions to the Dirac equation for a Coulomb potential in $\mathrm{D}+1$ dimensions, International Journal of Modern Physics E 11(4) (2002), 335-346.

DOI: https://doi.org/10.1142/S0218301302000879

[23] X. Y. Gu, Z. Q. Ma and S. H. Dong, The Levinson theorem for the Dirac equation in D + 1 dimensions, Physical Review A 67(6) (2003), 062715.

DOI: https://doi.org/10.1103/PhysRevA.67.062715 
[24] S. H. Dong, The Klein-Gordon equation with a Coulomb plus scalar potential in D dimensions, International Journal of Modern Physics E 13(3) (2004), 597-610.

DOI: https://doi.org/10.1142/S0218301304002338

[25] E. G. Kalnins, W. Miller Jr. and G. S. Pogosyan, The Coulomb-oscillator relation on $n$-dimensional spheres and hyperboloids, Physics of Atomic Nuclei 65(6) (2002), 1086-1094.

DOI: https://doi.org/10.1134/1.1490116

[26] E. G. Kalnins, W. Miller Jr. and G. S. Pogosyan, Exact and quasi-exact solvability of two-dimensional superintegrable quantum systems: I, Euclidean Space, arXiv:math$\mathrm{ph} / 0412035$.

[27] P. Winternitz and I. Yurdusen, Integrable and superintegrable systems with spin, Journal of Mathematical Physics 47(10) (2006); Article 103509.

DOI: https://doi.org/10.1063/1.2360042

[28] S. H. Dong and J. García-Ravelo, Exact solutions of the $s$-wave Schrödinger equation with Manning-Rosen potential, Physica Scripta 75(3) (2007), 307-309.

DOI: https://doi.org/10.1088/0031-8949/75/3/013

[29] A. D. Alhaidari, Systematic and intuitive approach for separation of variables in the Dirac equation for a class of noncentral electromagnetic potentials, Annals of Physics 320(2) (2005), 453-467.

DOI: https://doi.org/10.1016/j.aop.2005.07.001

[30] T. Cazenave and L. Vazquez, Existence of localized solutions for a classical nonlinear Dirac field, Communications in Mathematical Physics 105(1) (1986), $35-47$.

DOI: https://doi.org/10.1007/BF01212340

[31] F. Merle, Existence of stationary states for nonlinear Dirac equations, Journal of Differential Equations 74(1) (1988), 50-68.

DOI: https://doi.org/10.1016/0022-0396(88)90018-6

[32] Mikhael Balabane, Thierry Cazenave, Adrien Douady and Frank Merle, Existence of excited states for a nonlinear Dirac field, Communications in Mathematical Physics 119(1) (1988), 153-176.

DOI: https://doi.org/10.1007/BF01218265

[33] M. Balabane, Thierry Cazenave and Luis Vázquez, Existence of standing waves for Dirac fields with singular nonlinearities, Communications in Mathematical Physics 133(1) (1990), 53-74.

DOI: https://doi.org/10.1007/BF02096554

[34] M. J. Esteban and E. Sere, Existence of stationary solutions for the nonlinear Dirac equation and the Dirac-Poisson system, Comptes Rendus de l'Académie des Sciences: Série 1, Mathématiquet 319(11) (1994), 1213-1218. 
[35] M. J. Esteban and E. Sere, Stationary states of the nonlinear Dirac equation: A variational approach, Communications in Mathematical Physics 171(2) (1995), 323-350.

DOI: https://doi.org/10.1007/BF02099273

[36] Y. N. Ding and J. C. Wei, Stationary states of nonlinear Dirac equations with general potentials, Reviews in Mathematical Physics 20(8) (2008), 1007-1032.

DOI: https://doi.org/10.1142/S0129055X0800350X

[37] Y. Q. Gu, Space-time geometry and some applications of Clifford algebra in physics, Advances in Applied Clifford Algebras 28(4) (2018); Article 79.

DOI: https://doi.org/10.1007/s00006-018-0896-1

[38] Y. Q. Gu, The simplification of spinor connection and classical approximation, arXiv:gr-qc/0610001.

[39] Y. Q. Gu, Natural coordinate system in curved space-time, Journal of Geometry and Symmetry in Physics 47 (2018), 51-62. 